\title{
Improved Versions of Fielek Model for BCC Transition Metals
}

\author{
R. N. Khanna * and R. P. S. Rathore \\ Department of Physics, R. B. S. College, Agra (India)
}

Z. Naturforsch. 35a, 920-923 (1980); received June 3, 1980

\begin{abstract}
Three improved versions of the Fielek Model are developed and discussed. The first model uses a modified Bhatia scheme for the volume interaction in place of the Krebs scheme. The second and third model assumes the core-core couplings to be central pairwise effective upto first neighbours only. The second model describes the d-shell-d-shell interaction on the same lines as those adopted for core-core interactions, while the third model assumes these interactions to be of the many body type. Suitable equilibrium conditions for the second and the third model are developed.

These improved versions are employed to derive dispersion relations in tantalum. Comparison of these relations with-each other and also with the experimental findings leads to some important conclusions regarding the nature and range of the interactions coupling the various constituents of a bec transition metal like tantalum.
\end{abstract}

\section{Introduction}

The present communication deals with three improved and simplified versions of the Fielek model [1] for bcc transition metals. The first of these models differs from the original one [1] only in the description of the volume interactions. The Bhatia scheme [2] is modified to account (a) for the crystal anisotropy by using a realistic expression $[3,4]$ for the inference factor $G^{2}$, which is derived by summing up the total stress uniformly distributed over the whole atomic polyhedron, and (b) for the short range exchange and correlation effects $[5,6]$ associated with the local depletion in the density around each electron. This modified form of the Bhatia scheme saves a lot of computational effort, treats the electron in a quasi-free manner and removes the well known inconsistencies of the Krebs scheme [7] related with the screening parameter [8] and the electron gas [9].

The second and third model assumes the corecore interactions to be central pairwise effective upto next neighbours only. The second model describes the couplings among neighbouring d-shells to be expressible by the first two terms of the Taylor expansion of the coupling energy. The third model assumes these couplings to be of the many body type. The angular interactions developed by

\footnotetext{
* Department of Physics, R. E. I. College, Dayalbagh, Agra (India).

Reprint requests to Dr. R. P. S. Rathore, D-132 Kamla Nagar, Agra-282 005, Indien.
}

Clark et al. [10] seem to be suitable [11] three body interactions for this purpose. The volume interactions in these models are accounted for as in the first model.

It may be noted that the original Fielek model [1] and its subsequent applications [12-15] ignore the volume interactions for the system of core and conduction electrons. Moreover these studies lack an adequate, simple and consistent inclusion of an equilibrium condition. We have derived two different equilibrium constraints each for our second and third model. The interactions among core and d-shells are described in the manner of the original Fielek model [1].

Dispersion relations in tantalum are derived on the basis of these models. Theoretical predictions so obtained are compared mutually and with the experimental findings. This comparison leads to important conclusions regarding the nature and the range of the interactions coupling the various constituents of a bcc transition metal like tantalum.

\section{Formulations}

The secular equation solved for the dispersion frequency $(v)$ may be written as

$$
\left|D(\boldsymbol{q})-4 \pi^{2} m \nu^{2} I\right|=0,
$$

where $m$ is the mass of the core, $I$ is the unit matrix of $3 \times 3$ order and $D(\boldsymbol{q})$ is the dynamical matrix, whose elements can be expressed as 


$$
\begin{aligned}
& D_{a a}(\boldsymbol{q})=-\frac{8}{3} \beta_{1}\left(1-C_{a} C_{\beta} C_{\gamma}\right)-4 \beta_{2} S_{a}^{2}+K+A G(\boldsymbol{q})+K^{2} / N \\
& \left.\begin{array}{l}
D_{a \beta}(\boldsymbol{q})=-\frac{8}{3} \beta_{1} S_{a} S_{\beta} C_{\gamma} \\
D_{a \alpha}(\boldsymbol{q})=-\frac{8}{3}\left(\beta_{1}+2 \alpha_{1}\right)\left(1-C_{\alpha} C_{\beta} C_{\gamma}\right)+K+A G(\boldsymbol{q})+K^{2} / N \\
D_{a \beta}(\boldsymbol{q})=-\frac{8}{3}\left(\beta_{1}-\alpha_{1}\right) S_{a} S_{\beta} C_{\gamma}
\end{array}\right\} \begin{array}{l}
\text { model 1 }, \\
\text { and 3. }
\end{array}
\end{aligned}
$$

$A$ is the deformation parameter for the volume interactions among core and conduction electrons, $K$ is the parameter for core- $d$ shell bindings and $C_{a}=\cos \left(\frac{1}{2} a q_{a}\right), S_{a}=\operatorname{Sin}\left(\frac{1}{2} a q_{\alpha}\right)$, where $a$ is the lattice constant and $q_{a}$ the $\alpha$-th component of the phonon wave vector $(\boldsymbol{q})$. Also

$$
\alpha_{1}=\frac{1}{r_{1}}\left(\frac{\partial \omega_{1}}{\partial r_{1}}\right), \quad \beta_{1}=\frac{\partial^{2} \omega_{1}}{\partial r_{1}^{2}}, \quad \beta_{2}=\frac{\partial^{2} \omega_{2}}{\partial{r_{2}}^{2}},
$$

where $r_{1}$ and $r_{2}$ are the normal separations between the first and second neighbouring cores, respectively, and $\omega_{1}, \omega_{2}$ the central pairwise energies coupling them. $N$ is obtained by solving the determinant

$$
\left|D^{\prime}(\boldsymbol{q})-N I\right|=0,
$$

where $D^{\prime}(\boldsymbol{q})$ represent the dynamical matrix describing the interactions among the d-shells. For the first model,

$$
\begin{aligned}
& D_{\alpha \alpha}^{\prime}(\boldsymbol{q})=\frac{8}{3}\left(\beta_{1}^{\prime}\right) \cdot\left(1-C_{a} C_{\beta} C_{\gamma}\right)+K+A^{\prime} G(\boldsymbol{q}) \\
& D_{\alpha \beta}^{\prime}(\boldsymbol{q})=\frac{8}{3}\left(\beta_{1}^{\prime}\right) S_{a} S_{\beta} C_{\gamma} \\
& D_{\alpha \alpha}^{\prime}(\boldsymbol{q})=\frac{8}{3}\left(\beta_{1}{ }^{\prime}+2 \alpha_{1}{ }^{\prime}\right)\left(1-C_{\alpha} C_{\beta} C_{\gamma}\right)+K-A^{\prime} G(\boldsymbol{q}) \\
& D_{\alpha \beta}^{\prime}(\boldsymbol{q})=\frac{8}{3}\left(\beta_{1}^{\prime}-\alpha_{1}^{\prime}\right) S_{a} S_{\beta} C_{\gamma} \\
& \left.\begin{array}{l}
D_{\alpha \alpha}^{\prime}(\boldsymbol{q})=16 \gamma_{1}\left(1-C_{a} C_{\beta} C_{\gamma}\right)-2 \gamma_{1}\left(4 C_{2 a}-C_{2 \beta}-C_{2 \gamma}-2\right)+K-A^{\prime} G(\boldsymbol{q}) \\
D_{\alpha \beta}^{\prime}(\boldsymbol{q})=-8 \bar{\gamma}_{1} S_{a} S_{\beta} C_{\gamma}
\end{array}\right\} \text { model } 3 .
\end{aligned}
$$

Here $A^{\prime}$ is the deformation parameter for the volume interaction among d-shells and conduction electrons; $\gamma_{1}$ is the angular force constant for neighbouring $\mathrm{d}$-shells.

$$
\beta_{1}^{\prime}=\partial^{2} w_{1}^{\prime} / \partial r_{1}^{\prime 2}
$$

where $w^{\prime}$ is the central coupling energy for first and second neighbouring $d$-shells and $r_{1}^{\prime}$ is their equilibrium separation. According to adiabatic approximation,

$$
r_{1}=r_{1}^{\prime} \text {. }
$$

The dynamical matrix $G(\boldsymbol{q})$ describing the volume interaction may be written within the frame work of the Bhatia scheme as

$$
G_{a \alpha}(\boldsymbol{q})=q_{a}^{2} \Omega G^{2} /\left(a\left[1+q^{2} / K_{\mathrm{c}}^{2} \varepsilon(\boldsymbol{q})\right]\right)
$$

where $\Omega$ is the atomic volume. $G^{2}$, the inference factor, is evaluated using the expressions given in reference [4]. $K_{\mathrm{c}}$, the screening parameter is evaluated in the Bohm-Pine limit [16]. $\varepsilon(\boldsymbol{q})$, the dielectric function, is used to correct the screening parameter for its dependence on electron energy i. e.,

$$
\varepsilon(\boldsymbol{q})=\varepsilon_{\mathrm{H}}(\boldsymbol{q})[1-S(\boldsymbol{q})]
$$

where $\varepsilon_{\mathrm{H}}(\boldsymbol{q})$ is the usual Hartree dielectric function $S(\boldsymbol{q})$ is the correction term arising from the exchange and correlation effects associated with the 
depletion of the density around the electrons. This correction term is evaluated on the line of Vasistha and Singwi [17], whose fluctuation-dissipation theory satisfies the compressibility-sum-rule and leads to desirable value of the pair correlation function for small electron-separation.

For considering the equilibrium of the whole lattice, volume dependent energies associated with the cores and electrons are considered explicitly. The former energy is central pairwise while the details of the latter energy have recently been reported by our group [18, 19]. The required equilibrium constraints may be written as.

$$
\begin{array}{rlrl}
\alpha_{1}+\alpha_{1}^{\prime} & =-\frac{1}{2} a P_{\mathrm{e}} & & \text { model 2, } \\
\alpha_{1} & =-\frac{1}{2} a\left(P_{\mathrm{e}}+P_{\mathrm{d}}\right) & \text { model 3, }
\end{array}
$$

where $P_{\mathrm{e}}$ is the pressure associated with the conduction electrons and $P_{\mathrm{d}}$ is the corresponding pressure for the d-shell electrons. $P_{\mathrm{e}}$ (electron separation 3 ) and $P_{\mathrm{d}}$ (electron separation 2) are directly taken from [18] and [19], respectively. Further

$$
A=-a \Omega \frac{\partial \varrho_{\mathrm{e}}}{\partial \Omega} \text {. }
$$

\section{Calculations and Results}

The first and third model comprise six parameters whereas the second model comprises seven. For the first model $A=0$, and its remaining five parameters are calculated with the help of known values of the three elastic constants and two zone boundary frequencies, i. e. $v_{\mathrm{L}}[100]$ and $v_{\mathrm{T}}[100]$. The second model employs three elastic relations, Eqs. (13) and (15), $v_{\mathrm{T}}[100]$ and $v_{\mathrm{T} 1}[110]$. The third model uses three elastic relations, Eqs. (14) and (15) and $v_{\mathrm{T}}[100]$.

The input data and calculated model parameters for tantalum are shown in Table 1. Figure 1 depicts

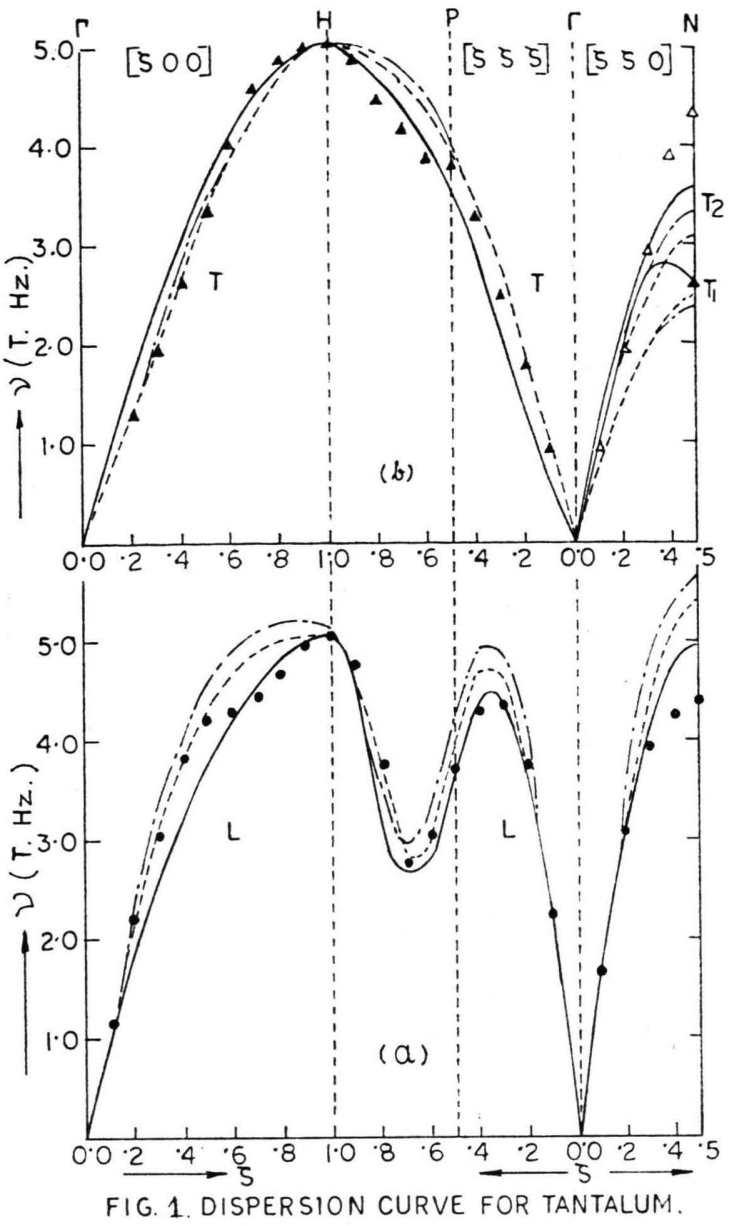

Fig. 1. Dispersion curves for tantalum (a) longitudinal (b) transverse modes, Experimental points $(\bullet, \mathbf{\Lambda}, \triangle)$ due to Woods [21]. Theoretical curves: $(-\cdot-\cdot-)$ model 1, $(-\longrightarrow)$ model $2,(-\ldots .$.$) model 3$.

the theoretically derived dispersion curves along with the experimental data $(\bullet, \Delta, \Delta)$ due to Woods

\begin{tabular}{|c|c|c|c|c|}
\hline \multirow[t]{2}{*}{ Input data } & \multirow[t]{2}{*}{ Reference } & \multicolumn{3}{|c|}{ Model parameters $\left(10^{4}\right.$ dyne $\left./ \mathrm{cm}\right)$} \\
\hline & & Model 1 & Model 2 & Model 3 \\
\hline $\left.\begin{array}{l}C_{11}=2.609 \\
C_{12}=1.574 \\
C_{44}=0.818\end{array}\right\} \begin{array}{l}10^{12} \\
\text { dyne } / \mathrm{cm}^{2}\end{array}$ & {$[20]$} & $\begin{array}{l}\beta_{1}=-9.837 \\
\beta_{2}=-1.7077 \\
\beta_{1}^{\prime}=5.7878\end{array}$ & $\begin{array}{l}\alpha_{1}=-0.2130 \\
\beta_{1}=-4.8279 \\
\alpha_{1}^{\prime}=0.6404\end{array}$ & $\begin{array}{l}\alpha_{1}=1.4524 \\
\beta_{1}=-5.4164 \\
\gamma_{1}=-0.5125\end{array}$ \\
\hline$a=3.3 \times 10^{-8} \mathrm{~cm}$ & & $K=82.4667$ & $\beta_{1}^{\prime}=-2.4864$ & $K=1311.968$ \\
\hline $\left.\begin{array}{l}v_{\mathrm{L}}[100]=5.03 \\
v_{\mathrm{T}}[100]=5.03 \\
v_{\mathrm{T}_{1}[110]}[1.63\end{array}\right\}$ T. & {$[21]$} & $A^{\prime}=2.4948$ & $\begin{array}{l}K=-2.8645 \\
A=\quad 0.2953 \\
A^{\prime}=\quad 5.6147\end{array}$ & $\begin{array}{ll}A= & 0.2953 \\
A^{\prime}= & 0.49\end{array}$ \\
\hline
\end{tabular}
[21].

Table 1. Input data and calculated model parameters for tantalum. 


\section{Conclusions}

It can be inferred from the curves (Fig. 1) that our model 3 reproduces the experimentally determined dispersion frequencies for the T-modes very well. For the L-modes model 2 seems to be closer to the experimental findings. The peculiar feature associated with $L[100]$ could not be explained by any of the present models, however our model 3 reproduces this branch with a reasonable degree of success.

[1] B. L. Fielek, J. Phys. F-5, 17 (1975).

[2] A. B. Bhatia, Phys. Rev. 97, 363 (1955).

[3] H. Bross and G. Bohn, Phys. Stat. Sol. 20, 277 (1967).

[4] V. Ramamurtty, Pramana 11, 233 (1978).

[5] A. K. Raja Gopal, Phys. Rev. A-6, 1239 (1972).

[6] H. Yashuhara, J. Phys. Soc. Japan 36, 361 (1974).

[7] K. Krebs, Phys. Rev. A 138, 143 (1965).

[8] R. P. S. Rathore, communicated to Acta Phys. Sc. Hungaracae.

[9] J. C. Upadhyaya, Phys. Rev. B-18, 2961 (1978).

[10] B. C. Clark, D. C. Gazis and R. F. Wallis, Phys. Rev. A-134, A 1486 (1964).

[11] R.P.S. Rathore, Acta Phys. Polonica A-55, 637 (1979).

[12] A. R. Jani and V. B. Gohel, J. Phys. F-6, L-25, (1976).
It can thus be concluded that the short range unpaired (angular) interactions among the neighbouring $d$-shells greatly influence the transverse vibrations, whereas longitudinal vibrations are dominantly governed by the central pairwise forces.

In general, the modified Bhatia scheme incorporating the equilibrium condition apparently explains the contribution due to the volume interactions in the phonon dispersion even at large wave vectors.

[13] B. P. Singh, L. P. Pathak, and M. P. Hemker, J. Phys. Soc. Japan 45, 484 (1978).

[14] B. P. Singh, L. P. Pathak, and M. P. Hemker, J. Phys. F-8, 2493 (1978).

[15] B. P. Singh and M. P. Hemker, Nuovo Cim. 50 B, 134 (1979).

[16] D. Bohm and D. Pines, Phys. Rev. 92, 609 (1953).

[17] P. Vasistha and K. S. Singwi, Phys. Rev. B-6, 875 (1972).

[18] R. P. S. Rathore and R. M. Agrawal, Phys. Stat. Sol. 97 (b), 597 (1980).

[19] R. N. Khanna and R. P. S. Rathore, to appear in Ind. J. Pure and Appld. Phys.

[20] C. Kittel, Introduction to Solid State Physics, p. 111, (1967).

[21] A. D. B. Woods, Phys. Rev. 136, 781 (1964). 